\title{
Salivary cortisol as a tool for physiological studies and diagnostic strategies
}

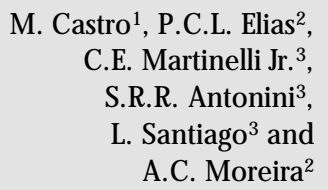

M. Castro ${ }^{1}$, P.C.L. Elias ${ }^{2}$, C.E. Martinelli Jr. ${ }^{3}$, S.R.R. Antonini ${ }^{3}$, L. Santiago ${ }^{3}$ and A.C. Moreira ${ }^{2}$

\author{
Divisão de Endocrinologia, Departamentos de ${ }^{1}$ Fisiologia, \\ ${ }^{2}$ Clínica Médica e ${ }^{3}$ Pediatria, Faculdade de Medicina de Ribeirão Preto, \\ Universidade de São Paulo, Ribeirão Preto, SP, Brasil
}

\section{Correspondence \\ A.C. Moreira \\ Departamento de Clínica Médica FMRP, USP \\ 14049-900 Ribeirão Preto, SP Brasil \\ Fax: + 55-16-633-1144 \\ E-mail: acmoreir@fmrp.usp.br \\ Presented at the First \\ International M eeting on Adrenal \\ Disease: Basic and Clinical \\ Aspects, Ribeirão Preto, SP, Brazil, \\ August 31-September 2, 1999. \\ Research supported by CNPq (No. 522219/96-9) and Hospital das Clínicas de Ribeirão Preto (HCFM RP-FAEPA). Publication supported by FAPESP.}

Received February 25, 2000 Accepted June 12, 2000

\section{Abstract}

Salivary cortisol is an index of plasma free cortisol and is obtained by a noninvasive procedure. We have been using salivary cortisol as a tool for physiological and diagnostic studies, among them the emergence of circadian rhythm in preterm and term infants. The salivary cortisol circadian rhythm in term and premature infants was established between 8 and 12 postnatal weeks. In the preterm infants the emergence of circadian rhythm was parallel to the onset of sleep rhythm. We also studied the use of salivary cortisol for screening for Cushing's syndrome (CS) in control and obese outpatients based on circadian rhythm and the overnight $1 \mathrm{mg}$ dexamethasone (DEX) suppression test. Salivary cortisol was suppressed to less than $100 \mathrm{ng} /$ dl after $1 \mathrm{mg}$ DEX in control and obese patients. A single salivary cortisol measurement at 23:00 $\mathrm{h}$ and again after $1 \mathrm{mg}$ DEX above the 90th percentile of the obese group values had sensitivity and specificity of 93 and 93\% (23:00 h), and 91 and 94\% (after DEX), respectively. The sensitivity improved to $100 \%$ when we combined both parameters. We also studied $11 \mathrm{CS}$ children and 21 age-matched primary obese children for whom salivary cortisol sensitivity and specificity were 100/95\% (23:00 h), and 100/95\% (1 mg DEX), respectively. Similar to adults, sensitivity and specificity of $100 \%$ were obtained by combining 23:00 $\mathrm{h}$ and $1 \mathrm{mg}$ DEX. The measurement of salivary cortisol is a useful tool for physiological studies and for the diagnosis of CS in children and adults on an outpatient basis.

\section{Introduction}

Salivary cortisol measurement by radioimmunoassay is an excellent index of plasma free cortisol concentration, independently of flow rate or transcortin fluctuations $(1,2)$. In addition, samples are obtained by noninvasive stress-free procedures, are easier to collect, even at home, and can obviate many
Key words

- Salivary cortisol

- Ontogeny

- Cushing's syndrome problems such as the lack of skilled personnel (3). Salivary samples may be collected many times a day and may provide information for physiological and diagnostic studies.

There are only a few previous longitudinal investigations using salivary cortisol $(4,5)$ and one using plasma cortisol (6) to study the mean age of appearance of the circadian rhythm in term infants. In addition, this in- 
formation is not available for premature infants. The ontogenetic maturation of the circadian sleep-wake rhythms in preterm and full-term infants has been widely accepted as the result of the length of exposure to environmental time cues, rather than neurologic maturity (7-9). These observations prompted us to compare the effects of prematurity on the temporal evolution of the circadian variation in salivary cortisol and on the daily rhythm of sleep and wakefulness in preterm infants.

Salivary cortisol may also be used to improve the diagnosis and differential diagnosis of Cushing's syndrome (CS), which is one of the most puzzling problems in endocrinology and remains controversial. Diagnostic difficulties can arise in distinguishing between CS from situations often referred to as pseudo-Cushing's states, including severe obesity, high blood pressure and depression among others (10-12), in which the clinical and biochemical evidence may be confusing. The difficulties in diagnosing CS are also present in childhood and adolescence. Excessive weight gain and growth retardation are the most common features and the typical phenotype with centripetal fat distribution and wasted appearance of the extremities due to increased protein catabolism is less frequent in childhood $(13,14)$. Therefore, the screening diagnosis applied to adult or pediatric patients with a clinical suspicion of hypercortisolism is highly dependent on biochemical confirmation. The diagnostic strategies are usually based on measurement of urinary free cortisol (UFC), a low dose or overnight dexamethasone (DEX) suppression test (15), and an evaluation of the diurnal variation of plasma cortisol $(10-12,16,17)$. The UFC has a sensitivity of about $95 \%$, but has the disadvantage of relying on patient management for proper collection, especially in young children. Because of the continuing need for improved noninvasive means of distinguishing pseudoCushing from CS, we used salivary cortisol measurement as a tool in screening for CS, evaluating hypercortisolism by the circadian rhythm and the overnight $1 \mathrm{mg}$ DEX suppression test for outpatients.

We shall attempt to review some of the novel findings, which could be used in physiological studies and as newer diagnostic techniques for CS in children and adult on an outpatient basis.

\section{Circadian rhythm ontogeny}

The mean age of appearance of the circadian rhythm of salivary cortisol secretion is from the second and third month in healthy term infants, as reported by us and others (4-6). These data suggest that circadian maturation of the hypothalamic-pituitary-adrenal axis occurs at an early postnatal age in normal infants. A previous study also reported an inverse relationship between gestational age and baseline plasma cortisol values in premature infants (18). We studied nine premature newborn infants with a gestational age of 31 to 34 weeks as evaluated by the somatic Capurro method (19). Salivary samples were obtained at weeks 2, 4, 8, 12, 16, 20 and 24 of postnatal life. Saliva samples were collected between 8:00 and 9:00 h (morning sampling) and between 21:00 and 22:00 $\mathrm{h}$ (night sampling) at the neonatal nursery and at the patient's home after discharge. The mothers were also instructed to record their infants' 24-h sleepwake activities on a sleep diagram during the $24 \mathrm{~h}$ preceding saliva collection (20).

The individual circadian rhythm emerged from 2 to 16 postnatal weeks. The median and mean for the appearance of this rhythm were 8 and 9.3 postnatal weeks, respectively, in this longitudinal study. The development of circadian cortisol rhythm obtained by pooling the results emerged in the group as a whole between 8 and 12 postnatal weeks. Similar findings were also observed by us for salivary cortisol rhythm in full-term babies using the same longitudinal protocol and salivary cortisol radioimmunoassay (5). 
These data clearly indicate that cortisol circadian rhythm starts between the second and third month of postnatal life both in term and preterm babies. These results suggest that the development of cortisol circadian rhythm in premature infants is not necessarily retarded and that gestational age (31 to 34 weeks) is not critical for the timing of emergence of the hypothalamic-pituitary-adrenal axis circadian activity (21).

The major action of the environment is to synchronize the circadian rhythms by frequent periodic signals, such as light-dark, rest-activity and sleep-wake cycles, food ingestion and social cues $(22,23)$. Therefore, we also evaluated the timing of the emergence of the 24-h rhythm of sleep and wakefulness in these premature babies. The onset of the circadian rhythm of sleep was detected from 8 postnatal weeks in this preterm group. This age is similar to those previously reported for preterm $(9.8 \pm 2$ weeks $)$ and term ( $8.7 \pm 2.7$ weeks) babies ( 8$)$. There was a parallelism between the appearance of such rhythm and the mean age at the onset of the salivary cortisol circadian rhythm. This similarity may indicate that the two parameters are intimately related or that this parallelism may simply be a coincidence. Our data suggest that the complex neuroanatomical pathways, including the hypothalamic nuclei of the cortisol circadian rhythm-generating system, are sufficiently developed between 31 and 34 post-conceptional weeks. At this time, the circadian rhythm is able to operate after delivery under the influence of internal and external synchronizers at a postnatal age similar to that of term infants.

\section{Salivary cortisol in Cushing's syndrome}

In a comparison among 30 normal volunteers (control), 18 obese patients, and 33 patients with CS basal nine o'clock salivary cortisol levels presented a substantial overlap in values. Consequently, the usefulness of morning salivary cortisol and morning plasma free cortisol is questionable and the measurement of these parameters cannot be recommended as a test for the differential diagnosis of hypercortisolism. The absence of a diurnal rhythm has been considered to be a hallmark of the diagnosis of CS $(24,25)$. In general, studies determined by spot or timed UFC between 22:00-23:00 h $(26,27)$, or a single sleeping midnight plasma cortisol (28), or salivary cortisol levels obtained at 23:00 $\mathrm{h}$ (29) have demonstrated that evening secretion is higher in CS than in normal subjects. The salivary cortisol values found in CS patients at 23:00 h were almost entirely outside the normal range, with a $15 \%$ overlap, compared to the values found in normal and obese groups. A cut-off salivary cortisol point above the 90th percentile of the normal control subjects at 23:00 h (168 $\mathrm{ng} / \mathrm{dl})$ produced a sensitivity $(100 \%)$ and a specificity $(88 \%)$ comparable to those described in two recent studies using a single sleeping midnight plasma cortisol determination in patients hospitalized for a period of at least $48 \mathrm{~h}(28,30)$. Our study further showed that the cut-off point for salivary cortisol above the 90th percentile $(280 \mathrm{ng} / \mathrm{dl})$ of the obese group values at 23:00 $\mathrm{h}$ produced $93.3 \%$ sensitivity and specificity, also similar to the values reported in another recent preliminary study (31).

The overnight $1 \mathrm{mg}$ DEX suppression test (32) is simple and reliable to differentiate cushingoid disorders from CS on an outpatient basis $(10,32)$. To improve its diagnostic accuracy and simplicity we tested whether substituting saliva for plasma cortisol determination may simplify the $1 \mathrm{mg}$ DEX suppression test. In fact, using as a criterion the cut-off point for salivary cortisol above the 90th percentile of the values for the normal control $(62 \mathrm{ng} / \mathrm{dl})$ and obese $(392 \mathrm{ng} / \mathrm{dl})$ groups, the test had sensitivity and specificity of $100 / 100 \%$ and $91.4 / 94.4 \%$, respectively. The overnight $1 \mathrm{mg}$ DEX suppression test presented sensitivity and speci- 
ficity similar to those of the 23:00 h salivary cortisol. We then evaluated the performance of the combination of both 23:00 h collection and overnight $1 \mathrm{mg}$ DEX suppression of salivary cortisol. In fact, when both tests from the same patients were combined, the sensitivity was higher $(100 \%)$ than that of either test performed individually. Our data indicate that bedtime $(23: 00 \mathrm{~h})$ collection and the overnight $1 \mathrm{mg}$ DEX salivary cortisol suppression test are sensitive markers for the diagnosis of CS.

We also studied 11 children with CS confirmed by standard laboratory investigation and surgical findings and 21 age-matched primary obese children as controls (33). In these groups salivary samples were collected at 23:00 h, and after an overnight DEX suppression test ( $20 \mu \mathrm{g} / \mathrm{kg}$ up to $1 \mathrm{mg}$ ). As we previously observed in adults with $\mathrm{CS}$, the sensitivity and specificity of salivary cortisol were 100 and $95 \%(23: 00 \mathrm{~h})$ and 100 and 95\% (after DEX), respectively. Sensitivity and specificity of $100 \%$ were obtained by combining the data for 23:00 h and after 1 mg DEX. Therefore, in adult or pediatric patients with $\mathrm{CS}$, we recommend the association of the two tests since the collection of saliva rather than blood is a practical, simple, and noninvasive outpatient alternative for the screening of hypercortisolism.

In conclusion, salivary cortisol measurement is a useful tool for physiological studies, is also simple and effective for the diagnosis of CS, both in children and adults, and has the advantage of being performed on an outpatient basis. These issues are of practical clinical importance in recommending salivary cortisol as the best and simplest means to evaluate patients with suspected CS.

\section{References}

1. Walker RF, Riad-Fahmy D \& Read GF (1978). Adrenal status assessed by direct radioimmunoassay of cortisol in whole saliva or parotid saliva. Clinical Chemistry, 24: 1460-1463.

2. Umeda T, Hiramatsu R, Iwaoka T, Shimada T, Miura F \& Sato T (1981). Use of saliva for monitoring unbound free cortisol levels in serum. Clinica Chimica Acta, 110: 245-253.

3. Luthold WW, Marcondes JAM \& Wajchenberg BL (1985). Salivary cortisol for the evaluation of Cushing's syndrome. Clinica Chimica Acta, 151: 33-39.

4. Price DA, Close GC \& Fielding BA (1983). Age of appearance of circadian rhythm in salivary cortisol values in infancy. Archives of Disease in Childhood, 58: 454-456.

5. Santiago LB, Jorge SM \& Moreira AC (1996). Longitudinal evaluation of the development of salivary cortisol circadian rhythm in infancy. Clinical Endocrinology, 44: 157-161.

6. Mantogos S, Moustogiannis A \& Vagenakis AG (1998). Diurnal variation of plasma cortisol levels in infancy. J ournal of Pediatric Endocrinology and Metabolism, 11: 549-553.

7. Mirmiran M \& Kok J H (1991). Circadian rhythms in early human development.
Early Human Development, 26: 121-128.

8. MCMillen IC, Kok J SM, Adamson TM, Deayton J M \& Nowak R (1991). Development of circadian sleep-wake rhythms in preterm and full-term infants. Pediatric Research, 29: 381-384.

9. Shimada $M$, Segawa $M$, Higurashi $M$ \& Akamatsu H (1993). Development of the sleep and wakefulness rhythm in preterm infants discharged from a neonatal care unit. Pediatric Research, 33: 159-163.

10. Crapo L (1979). Cushing's syndrome: a review of diagnostic tests. Metabolism, 28: 955-977.

11. Bertagna X, RauX-Demay MC, Guilhaume B, Girard F \& Luton J P (1995). Cushing's disease. In: Melmed S (Editor), The Pituitary. 1st edn. Blackwell Science, Cambridge, England, 478-545.

12. Trainer PJ \& Grossman A (1991). The diagnosis and differential diagnosis of Cushing's syndrome. Clinical Endocrinology, 34: 317-330.

13. Magiakou MA, Mastorakos G, Oldfield EH, Gomes MT, Doppman J L, Cutler J r GB, Nieman LK \& Chrousos GP (1994). Cushing's syndrome in children and adolescents: presentation, diagnosis and therapy. New England J ournal of Medicine, 331: 629-636.
14. Weber A, Trainer PJ, Grossman AB, Afshar F, Medbak S, Perry LA, Plowman PN, Ress LH, Besser GM \& Savage MO (1995). Investigation, management and therapeutic outcome in 12 cases of childhood and adolescent Cushing's syndrome. Clinical Endocrinology, 43: 19-28.

15. Cronin C, Igoe D, Duffy MJ , Cunningham SK \& McKenna TJ (1990). The overnight dexamethasone test is a worthwhile screening procedure. Clinical Endocrinology, 33: 27-33.

16. Orth DN (1991). Differential diagnosis of Cushing's syndrome. New England J ournal of Medicine, 325: 957-995.

17. Findling J W \& Doppman J L (1994). Biochemical and radiological diagnosis of Cushing's syndrome. Endocrinology and Metabolism Clinics of North America, 23: 511-537.

18. Scott SM \& Watterberg KL (1995). Effect of gestational age, postnatal age, and illness on plasma cortisol concentrations in premature infants. Pediatric Research, 37: 112-116.

19. Capurro H, Konichevzky S, Fonseca D \& Caldeyro-Barcia P (1978). A simplified method for diagnosis of gestational age in the neonate newborn infants. J ournal of Pediatrics, 93: 120-122. 
20. Antonini SRR, J orge SM \& Moreira AC (2000). The emergence of salivary cortisol circadian rhythm and its relationship to sleep activity in preterm infants. Clinical Endocrinology, 52: 423-426.

21. J ett PL, Samuels MH, McDaniel PA, Benda GI, LaFranchi SH, Reynolds J W \& Hanna CE (1997). Variability of plasma cortisol levels in extremely low birth weight infants. J ournal of Clinical Endocrinology and Metabolism, 82: 2921-2925.

22. Krieger DT (1979). Rhythms in CRF, ACTH and corticosteroids. In: Krieger DT (Editor), Endocrine Rhythms. Raven Press, New York

23. Leal AMO \& Moreira AC (1997). Food and the circadian activity of the hypothalamicpituitary-adrenal axis. Brazilian J ournal of Medical and Biological Research, 30: 1391-1405.

24. Boyar RM, Witkin M, Carruth A \& Ramsey J (1979). Circadian cortisol secretory rhythms in Cushing's disease. J ournal of Clinical Endocrinology and Metabolism, 48: 760-765.

25. Liu J H, Kazer RR \& Rasmussen DD (1987). Characterization of the twentyfour hour secretion patterns of adrenocor- ticotropin and cortisol in normal women and patients with Cushing's disease. J ournal of Clinical Endocrinology and Metabolism, 64: 1027-1035.

26. Contreras LN, Hane S \& Tyrrell J B (1986). Urinary cortisol in the assessment of pituitary-adrenal function. Utility of 24 hour and spot determinations. J ournal of Clinical Endocrinology and Metabolism, 62: 965-969.

27. Laudat $\mathrm{MH}$, Billaud L, Thomopoulos $\mathrm{P}$, Vera O, Yllia A \& Luton J P (1988). Evening urinary free cortisol: a screening test in Cushing's syndrome and incidentally discovered adrenal tumors. Acta Endocrinologica, 119: 459-464.

28. Newell-Price J , Trainer P, Perry L, Wass J , Grossman A \& Besser M (1995). A single sleeping midnight cortisol has $100 \%$ sensitivity for the diagnosis of Cushing's syndrome. Clinical Endocrinology, 43: 545550.

29. Castro M, Elias PCL, Quidute ARP, Halah FPB \& Moreira AC (1999). Outpatient screening for Cushing's syndrome: the sensitivity of the combination of circadian rhythm and overnight dexamethasone suppression salivary cortisol tests. J our- nal of Clinical Endocrinology and Metabolism, 84: 878-882.

30. Papanicolaou DA, Yanovski JA, Cutler GBJ , Chrousos GP \& Nieman LK (1998). A single midnight serum cortisol measurement distinguishes Cushing's syndrome from pseudo-Cushing's states. J ournal of Clinical Endocrinology and Metabolism, 83: 1163-1167.

31. Papanicolaou DA, Yanovski J A, Cutler GBJ , Chrousos GP \& Nieman LK (1994). A single midnight cortisol measurement discriminates Cushing's syndromes from pseudo-Cushing's states. Proceedings of the 76th Meeting of the Endocrine Society, J une 15-18, Annaheim, CA, 518 (Abstract).

32. Nugent CA, Nichols T \& Tyler FH (1965). Diagnosis of Cushing's syndrome. Single dose dexamethasone suppression test. Archives of Internal Medicine, 115: 602605.

33. Martinelli CE, Sader SL, Oliveira EB Daneluzzi J C \& Moreira AC (1999). Salivary cortisol for screening of Cushing's syndrome in children. Clinical Endocrinology, 51: 67-71. 